\title{
Ship Main Engine Rotation Rate Measured Based on Opto-elemetronic Method
}

\author{
Guoxiong Huang ${ }^{1, a}$, Chao Chen ${ }^{1, b}$ and Yongming $\mathrm{Nie}^{1, \mathrm{c}^{*}}$ \\ ${ }^{1}$ China Satellite Maritime Tracking and Controlling Department, Jiangsu, Jiangyin, China, 214431 \\ a290505026@qq.com, byimonie@163.com, cnwy1986@163.com \\ * The corresponding author
}

Keywords: Optical measurement; Rotationrate; Pulse counting; Protothreads

\begin{abstract}
Based on thorough analysis of the integrated information transmission platform, a setup is demonstrated that can measure ship main engine rotating rate based on opto-elemetronic method, which includes opto-elemetronic sensor block, data collecting terminal, display terminal and power supply module. Then, combing V.35 channel integrated information transmission platform with protothreads mutiple-thread technology, the method to configure forward and reverse V.35 transmission channel has been developed, which can effectively reduce the demodulation error caused by signal distortion, simplify the wiring of the system, and enhances the deployment flexibility of time code terminal.
\end{abstract}

\section{Introduction}

Ship main engine rotation rate measuring is very important for ship secure, which refers to the measurement of the rotational speed of the mechanical rotational components, generally revolutions per minute or revolutions per second being its unit [1,2]. Mostly, speed measurement is mainly measuring mechanical shaft. Speed can not direct obtain and the measurement process needs to convert the speed for measuring signals. According to the characteristics and measuring principle of the signal, speed measurement technology can be divided into analog measurement technique and digital measurment technique [3-5]. The analog measuring technique is a technique which converts the rotational speed into the analog quantity and then carries out the measurement. The advantage is that the structure is simple, the connection is reliable, and the design is flexible. Drawback is the low precision, poor anti-interference, not suitable for long-distance transmission. Moreover, tachogenerator is physically connected with the rotating shaft, increasing the measured shaft rotational load and affecting the measured shaft rotation. Digital measuring technology which converts the rotating speed anolog quantity to digital quantity uses digital pulse signal as the signal measurement $[6,7]$. The advantages includ strong interference resistance, high measuring precision, high reliability, not affecting the measured shaft rotation and long-distance transmission does not affect the measurement accuracy. The disadvantage include that the system is complex and the sensor mounting needs higher requirements. The key technology of digital measuring technology is its sensor. According to different working principle of speed sensor, the commonly used speed sensors include magnetoelectric speed sensor, Hall Effect speed sensor and photoelectric speed sensor [8-10].

According to the analysis above, opto-elemetronic method is used to measure the rotation speed, which is non-contact speed measuring method. Photoelectric speed sensor does not connect with the object to be measured and has no additional load, so photoelectric speed sensor with less measurement error and higher precision and the measurement capability is good. Moreover, photoelectric speed sensor can be used in optical fiber package and can measure small objects. The photoelectric speed sensor has stable operation, good reliability and high precision, which can meet the requirements of users. 


\section{Theories}

The output pulse signal of the sensor represents the speed. According to the principle of measurement, speed measurement algorithms include pulse counting method and pulse cycle measurement method.

Pulse Counting Measuring Principle. The pulse counting method can be realized by counting the pulse in unit time, the principle of which is shown in Fig. 1.

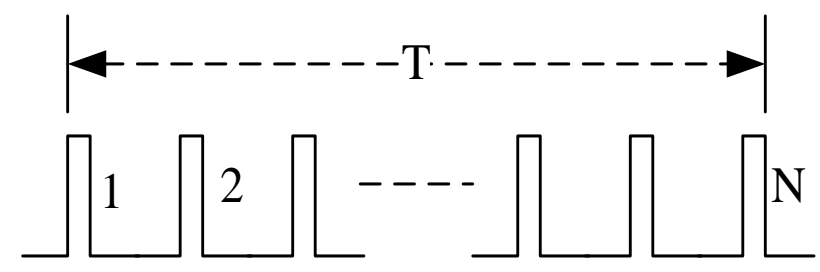

Figure 1. Schematic diagram of pulse counting method

Combining with the cooperative target such as gear number expressed with letter $\mathrm{n}$, the rotation speed can be written as follwing.

$$
v_{r}=f \times \frac{60}{n}=\frac{N}{T} \times \frac{60}{n}=N \times \frac{60}{T \times n}
$$

Analysis indicates that cycle counting method is determined by the interaction of rotation speed measuring resolution and gear number. Theoretical caculating results indicate that the longer the counting cycle is, the higher the measurement accuracy is. However, if the counting period is too long, the measuring data update frequency is too low, which results in poor real-time and low practicality. So usually a combination of considering the resolution and other factors is properly chosen to obtain a reasonable scheme. The measurement method of pulse technique is the average speed in the period $\mathrm{T}$. The system measurement error is large with rapid speed changes, which can not real-time giving large instantaneous changes.

Measurement Principle Based on Pulse Period. By measuring the time interval between the adjacent pulses with period $\mathrm{T}$, the pulse period measurement method can be accomplished and the principle of the pulse counting method is shown in Fig. 2.

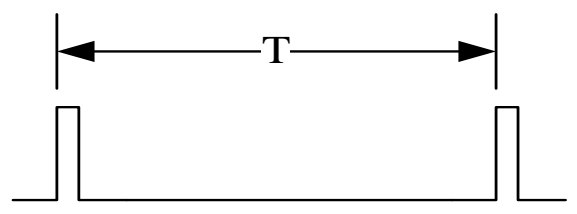

Figure 2. Schematic diagram of pulse period measurement method

According to gear number $\mathrm{n}$, the rotation speed can be obtained by transform indicated as the following formula.

$$
v_{r}=f \times \frac{60}{n}=\frac{1}{T} \times \frac{60}{n}=\frac{60}{T \times n}
$$

Pulse cycle measurement method is based on direct measurement of the pulse cycle, which can reveal transient state with high real-time performance and high measurement precision. Limited to the machining accuracy of gear and shaft jitter caused by the sensor spacing changing, a large jitter error is generated. In order to reduce the error influence, in general, average value of multiple cycles is used to calculate the speed. Number of cycles increasing in a certain extent reduces the measurement real-time, but can through reasonable value to achieve the real-time and precision of the measurement of the equilibrium for speed change faster system using pulse cycle measurement method, which is appropriate. 


\section{System Designations}

A setup is designed that can measure ship main engine rotating rate based on opto-elemetronic method that includes opto-elemetronic sensor block, data collecting terminal, display terminal and power supply module, which is shown in Fig. 3.

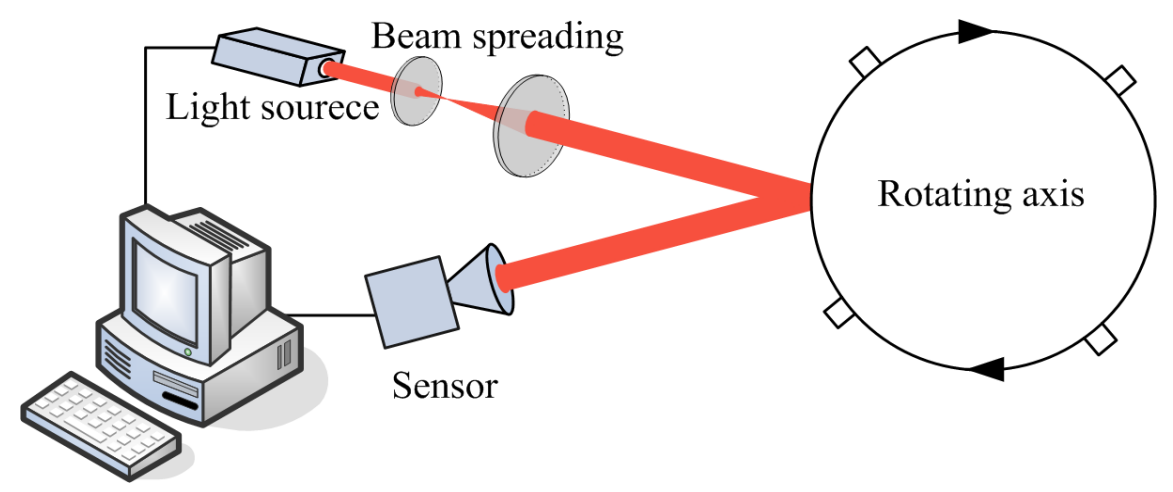

Figure 3. Experimental system, two lenses constituting a beam expander and the computer dealing with the collecting data and giving the results

Main engine speed measurement system needs to determine forward direction and opposite direction in addition to speed measurement values. When using a single sensor just one representative speed pulse bursts can be obtained. So another sensor is required to determine the result and two sensors installed position staggered a certain distance, which can make the two pulse trains generate a phase difference. By detecting the phase difference the result can be obtained. The principle is shown in Fig. 4. In order to facilitate the installation, the rotating speed sensor is selected in a single shell body to package two channel sensors, which is convenient for installation and debugging and can simplify the installation structure design.

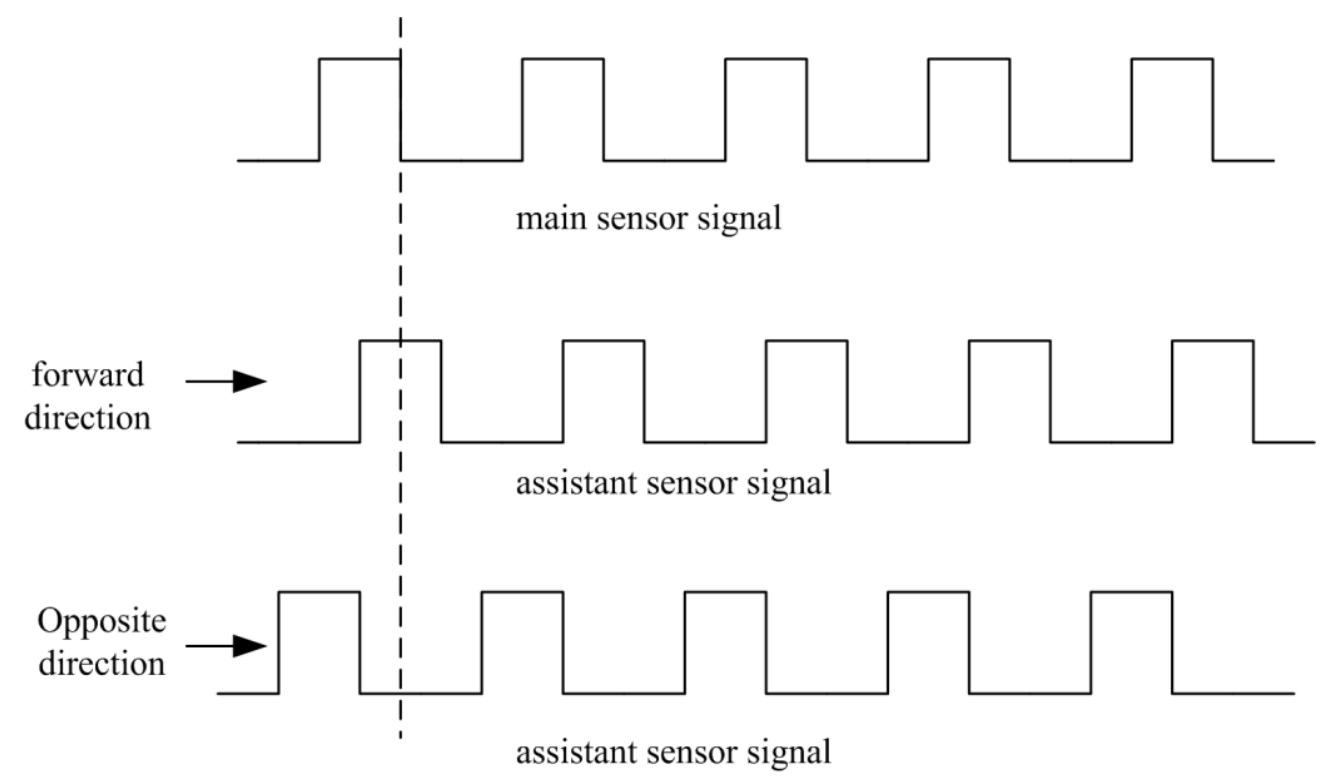

Figure 4. Schematic diagram of rotation detection

Take main speed sensor signal edge as a reference, because of existing difference between the output signals' phase, we can get two different sensor signal. When the main engine revolves forward, the main sensor signal phase is advancing the assistant sensor signal. When the main engine revolves opposite, the main sensor signal phase is falling behind the assistant sensor signal. The information can be obtained when there is a decline in the main sensor with forward for high level and inversion for low level. 
According to the functions of data acquisition terminal, data acquisition terminal external interfaces mainly including left main sensor interface, right main sensor interface, network interface, two display interfaces and a power supply interface. Considering the reliability and stability of the system design, the mature and reliable MCU embedded system designin is used, and data acquisition terminal block diagram is as shown in Fig. 5.

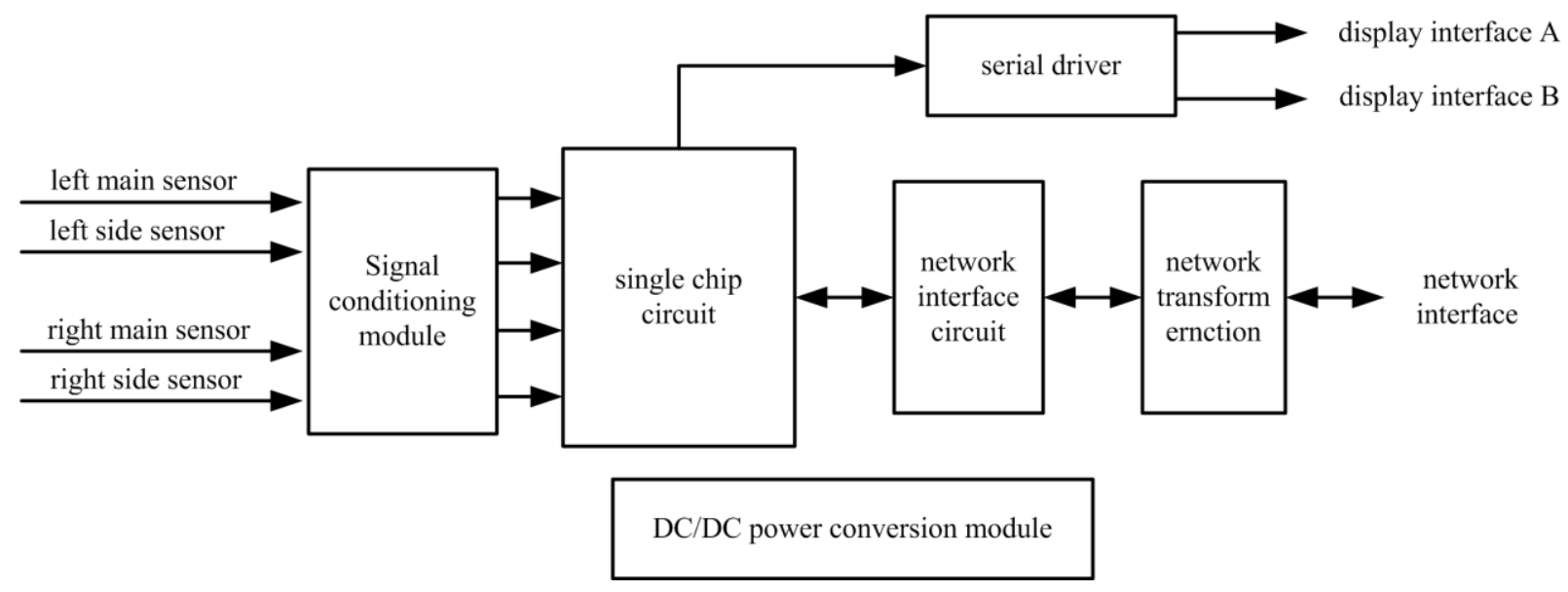

Figure 5. Data acquisition terminal block diagram

Data acquisition terminal mainly finished the engine speed detection, displayed communication protocol processing, and dealt with network communication protocol processing function, the work flow of which can be shown as following.

Firstly, after terminal is powered, initialize the input output port, serial port, input interrupt and timer settings. Secondly, set IP address and MAC address and other network parameters to complete the network initialization. Subsequently, restart the watchdog timer and interrupt. In this case, the interrupt system began to work.

Then initialize the display threads and program start its work into the main loop. In the main loop in order to run threads and reset the watchdog timer is displayed. Speed detection is completed in the interrupt program. Timer interrupt starts according to sampling period and the timing interval of the input pulse counting interrupt occurs when sampling time expires. Reading the counting values of transform coefficients speed data can be obtained. Based on rising time in the main sensor pulse edge interrupt, the information is obtained according to the auxiliary sensor pulse level.

\section{Experimental Results}

Based on the setup we designed, which includes opto-elemetronic sensor block, data collecting terminal, display terminal and power supply module, we got the experimental results. The test results show that the time delay compensation is less than the minimum 3 us and the maximum value is greater than $10 \mathrm{~ms}$. If the compensation of the transmission delay, it is less than 4 us. Moreover, the system can work on more than 5000 hours.

\section{Conclusions}

Based on thorough analysis of the integrated information transmission platform, the composition and characteristics of the time delay in V.35 channel is analyzed.The adaptive time delay compensation algorithm has been developed. Based on AVR MCU and Protothreads mutiple-thread technology, the adaptive time delay compensation terminal is design. Automatic measurement and adaptive compensation of the transmission channel transmission delay is realized. The scheme does not need to change the time code terminal, using V.35 channel of integrated information transmission platform to transmission high precision time information can effectively reduce the demodulation error caused by 
signal distortion, simplify the wiring of the system, and enhances the deployment flexibility of time code terminal.

\section{References}

[1] D. W. Handani and M. Uchida. Simulation on optimum operation of ship main engine support system by using System Dynamics, IEEE International Conference on Industrial Engineering and Engineering Management, 2012: 1935-1939.

[2] L. You, X.S. Wang and Z.S. Huang. Shaft Orientation Error Compensation of Single-axis Rotation INS in Ship Deformation Measurement System, Chinese Control \& Decision Conference, 2014, 54(8):1327-1336.

[3] W.U. Zongqin, P. Liu, X. Wang and et al. Research on the Measuring Method of the Rotation Speed of a Rotary Table Based on CCD Camera, Journal of Changchun University of Science and Technology, 2015, 1: 45-49.

[4] M. Baszynski, S. Pirog. A Novel Speed Measurement Method for a High-Speed BLDC Motor Based on the Signals from the Rotor Position Sensor, IEEE Transactions on Industrial Informatics, 2014, 10(1): 84-91.

[5] J.M. Lee, Y. Hwang. A novel online rotor condition monitoring system using fiber Bragg grating (FBG) sensors and a rotary optical coupler, Measurement Science \& Technology, 2008, 19(6):579-588.

[6] C. He. Test and Analysis of Digital Measuring System for Rotating Speed, Journal of Suzhou Institute of Silk Textile Technology, 1998, 02: 21-24.

[7] J. Zhang. A Novel Approach to Measuring Rotating Speed, Journal of Beijing Institute of Petro-Chemical Technology, 2012, 03:125-127.

[8] G.S. Shi, W.G. Zhang. Research and application of non-contact photoelectric speed sensor, Journal of Transducer Technology, 2002, 21(10):37-39.

[9] Murugan. Enhancing the Linearity Characteristics of Photoelectric Displacement Sensor Based on Extreme Learning Machine Method, Photonic Sensors, 2015, 5(1):24-31.

[10] Y. Su, Q. Wang, F. Yan and et al. Subdivision Error Analysis and Compensation for Photoelectric Angle Encoder in a Telescope Control System, Mathematical Problems in Engineering, 2015, 2015(16):1-9. 